\title{
sciendo
}

ISSN: $1231-4005$

e-ISSN: $2354-0133$

DOI: $10.2478 /$ kones-2019-0086

\section{SIMULATIONS OF THE INFLUENCE OF THE HEAT FLUX AT THE SHAFT SURFACE OF THE CONICAL SLIDE BEARING ON ITS HYDRODYNAMIC LUBRICATION AND OPERATING PARAMETERS}

\author{
Adam Czaban, Andrzej Miszczak \\ Gdynia Maritime University, Faculty of Marine Engineering \\ Morska Street 81-87, 81-225 Gdynia, Poland \\ tel.: $+48585586304,+48585586348$, fax: +48585586399 \\ e-mail:a.czaban@wm.umg.edu.pl,a.miszczak@wm.umg.edu.pl
}

\begin{abstract}
The aim of this work is to investigate, how in the adopted model of hydrodynamic lubrication of a conical slide bearing, the change of the heat flux value at the bearing shaft, affects bearing operating parameters. In this research, the authors use, the known from the literature, Reynolds type equation, describing the stationary hydrodynamic lubrication process of a conical slide bearing. The analytical, solutions, that determine the components of the lubricating oil velocity vector and the equation (analytical solution of energy equation) determining the threedimensional temperature distribution in the lubrication gap, was also adopted from previous works. In order to obtain numerical solutions, the Newton's method was used, and the derivatives in the Reynolds type equation were approximated by the finite differences. An application of the method of subsequent approximations allowed considering the influence of temperature, pressure and shearing rate on the viscosity of lubricating oil. The considerations were performed by adopting the Reynolds condition of the hydrodynamic oil film. It was tested, how the assumed value of the heat flux on the bearing shaft surface affects the values of the obtained operating parameters, i.e. the transverse and longitudinal component of the load carrying capacity, friction force and coefficient of friction.
\end{abstract}

Keywords: hydrodynamic lubrication, slide bearing, conical bearing, heat conduction, pressure distribution, viscosity

\section{Introduction}

One of the designed operating parameters of the hydrodynamic slide bearing is the viscosity of a lubricating oil. The viscosity of oils depends on the temperature, pressure and shear rate. Neglecting the influence of other factors (e.g. adhesive forces, magnetic field, electric field, oil aging), it can be written, that the viscosity of lubricating oil $\eta_{p}=\eta_{p}(T, p, \dot{\gamma})$ (where: $T-$ temperature in $[\mathrm{K}], p$ - pressure in $[\mathrm{Pa}], \dot{\gamma}$ - shear rate in $\left[\mathrm{s}^{-1}\right]$ ).

In the hydrodynamic lubrication theory, using the equations of the momentum conservation law and the stream continuity equation, along with the simplification for a thin lubricating layer of the slide bearing (in particular, omitting changes in hydrodynamic pressure towards the height of the lubrication gap), a Reynolds-type equation is derived $[1,5,6]$ (in which, the viscosity value occurs), which general analytical solution is unknown. However, it can be used in numerical calculations to determine the hydrodynamic pressure distribution in the bearing lubrication gap. By using the method of successive approximations, the calculations taking into account the effect of pressure on viscosity, can be carried out. There are also known, the analytical solutions of the simplified momentum equations, defining the components of oil velocity vector in a thin lubricating layer $[1,5,6]$. By using these functions, the three-dimensional distribution of the shear rate values in the lubrication gap, can be determined, and then its impact on the viscosity. The determination of three-dimensional temperature distribution in the lubrication gap, and taking into account its effect on viscosity, requires solving the equation of the energy conservation law. In this article, the authors use a known analytical solution of a simplified energy equation for a thin 
lubricating layer of a stationary process of lubrication $[1,5,6]$. For the solution of this problem and determination of the integration constants, two boundary conditions are required. In the adopted analytical solution of the energy equation, it was assumed, that there is a known, constant temperature at the surface of the shaft. The second boundary condition was that a (constant) heat flux is known at the surface of the shaft. The value of the heat flux at the shaft surface adopted in the simulations, influences the calculated temperature distribution in the lubrication gap, and changes the viscosity of the lubricating oil. The aim of this work is to investigate, how in the adopted model of hydrodynamic lubrication of a conical slide bearing, the change of the heat flux value at the bearing shaft, affects bearing operating parameters.

\section{Bearing model}

In Fig. 1 is shown the geometry of the investigated slide conical bearing.

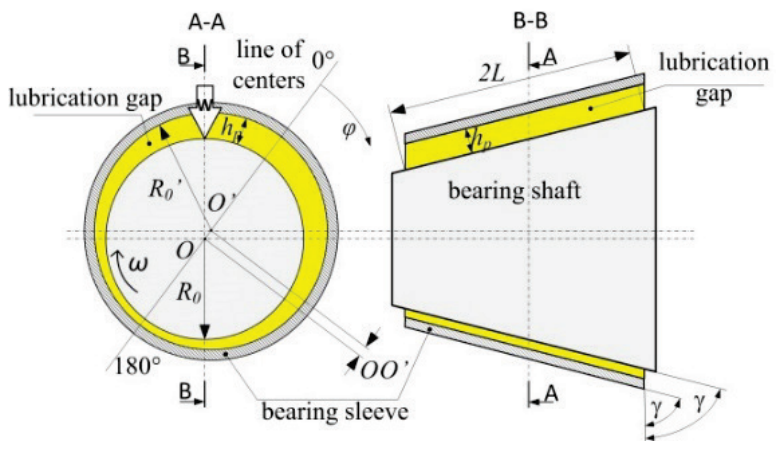

Fig. 1. The geometry of concerned slide conical bearing - radial and axial cross-section

The bearing, with a full wrap angle, which operates in a steady state, was considered in this research. The assumptions adopted in the research, are:

- the shaft rotation axis is parallel to the axis of the sleeve,

- the bearing surfaces are smooth, rigid and without deformations,

- the bearing sleeve is stationary and the hydrodynamic pressure is generated due to the rotation of the bearing shaft with constant speed $\omega$ [rpm] (no vibrations, constant load),

- the flow of incompressible, non-Newtonian lubricant is laminar and non-isothermal,

- the pressure at the edges of the wedge is equal to the ambient pressure, where the end of the lubricating wedge in the circumferential direction is determined by the Reynolds $[1,3,5]$ (Swift - Steiber) boundary condition:

$$
\left.\frac{\partial p}{\partial \varphi}\right|_{\varphi=\varphi_{k}}=0
$$

- at the bearing shaft and sleeve surfaces, the components of the velocity of the oil layer have the same value as the surface to which they adhere,

- the temperature on the entire surface of the bearing shaft is constant; the temperature at the surface of the sleeve is unknown, and a constant value of the heat flux $q_{c}\left[\mathrm{~W} / \mathrm{m}^{2}\right]$ is exchanged between the surface of the shaft and the lubricating oil.

The determination of the hydrodynamic pressure distribution in the lubrication gap consisted in the numerical solution of the dimensionless Reynolds type equation [1], which can be written (in the adopted conical coordinate system) in the following form:

$$
\Theta \frac{\partial}{\partial \varphi}\left(\int_{0}^{h_{p 1}} \Gamma_{m} \mathrm{~d} y_{1}-h_{p 1}\right)=\left(\frac{1}{\Theta} \frac{\partial^{2} p_{1}}{\partial \varphi^{2}}+\frac{\cos \gamma}{L_{1}} \frac{\partial p_{1}}{\partial x_{1}}+\frac{\Theta}{L_{1}^{2}} \frac{\partial^{2} p_{1}}{\partial x_{1}^{2}}\right)_{0}^{h_{p 1}} \Gamma_{d} \mathrm{~d} y_{1}-\frac{\rho_{1} \operatorname{Re} \psi \cos \gamma}{L_{1}} \frac{\partial}{\partial x_{1}}\left(\int_{0}^{h_{p 1}} \Gamma_{c} \mathrm{~d} y_{1}\right)
$$


where the following dimensionless values and functions have been introduced: $\Theta=1+L_{1} x_{1} \cos \gamma_{\text {, }}$ $L_{1}=L R_{0}^{-1}, y_{1}=y \varepsilon_{s}^{-1}$, where $0 \leq y_{1} \leq h_{p 1}, x_{1}=x L^{-1}$, where $-1 \leq x_{1} \leq 1, p_{1}=p p_{0}{ }^{-1}$, where $p_{0}=U_{0} \eta_{0} R_{0}^{-1} \psi^{-2}, U_{0}=\omega R_{0}, \eta_{0}[\mathrm{~Pa} \cdot \mathrm{s}]$ is a characteristic value of viscosity; the relative radial clearance $\psi=\varepsilon_{s} R_{0}^{-1} \sim 10^{-3}$. The dimensionless height of the lubrication gap $h_{p 1}=h_{p} \varepsilon_{s}^{-1}$, is determined by the function $[1,5]$ :

$$
h_{p 1}(\varphi)=(1+\lambda \cos \varphi)(\sin \gamma)^{-1},
$$

where the relative eccentricity $\lambda=O O^{\prime} / \varepsilon_{s}$. Re is the Reynolds number defined as: $\operatorname{Re}=\varepsilon_{s} U_{0} \rho_{0} \eta_{0}{ }^{-1}$, where $\rho_{0}\left[\mathrm{~kg} / \mathrm{m}^{3}\right]$ is a characteristic value of density.

The Eq. (2) also introduces the functions $\Gamma_{m}\left(\varphi, y_{1}, x_{1}\right), \Gamma_{d}\left(\varphi, y_{1}, x_{1}\right), \Gamma_{c}\left(\varphi, y_{1}, x_{1}\right)$, where:

$$
\begin{gathered}
\int_{0}^{h_{p_{1}}} \Gamma_{m} \mathrm{~d} y_{1}=\left(\int_{0}^{h_{p_{1}}} \int_{0}^{y_{1}} \frac{1}{\eta_{1}} \mathrm{~d} y_{1} \mathrm{~d} y_{1}\right)\left(\int_{0}^{h_{p_{1}}} \frac{1}{\eta_{1}} \mathrm{~d} y_{1}\right)^{-1}, \\
\int_{0}^{h_{p_{0}}} \Gamma_{d} \mathrm{~d} y_{1}=\int_{0}^{h_{0}} \int_{0}^{y_{1}} \frac{y_{1}}{\eta_{1}} \mathrm{~d} y_{1} \mathrm{~d} y_{1}-\left(\int_{0}^{h_{p_{1}}} \Gamma_{m} \mathrm{~d} y_{1}\right)\left(\int_{0}^{h_{m}} \frac{y_{1}}{\eta_{1}} \mathrm{~d} y_{1}\right), \\
\int_{0}^{h_{p}} \Gamma_{c} \mathrm{~d} y_{1}=\left(\frac{1}{\Theta} \frac{\partial p_{1}}{\partial \varphi}\right)^{2} \int_{0}^{h_{p_{p}}} \Gamma_{1} \mathrm{~d} y_{1}-2 \frac{\partial p_{1}}{\partial \varphi} \int_{0}^{h_{m}} \Gamma_{2} \mathrm{~d} y_{1}+\Theta^{2} \int_{0}^{h_{p}} \Gamma_{3} \mathrm{~d} y_{1},
\end{gathered}
$$

where $\eta_{1}=\eta_{p} \eta_{0}^{-1}$, while integrals of functions $\Gamma_{1}\left(\varphi, y_{1}, x_{1}\right), \Gamma_{2}\left(\varphi, y_{1}, x_{1}\right), \Gamma_{3}\left(\varphi, y_{1}, x_{1}\right)$ are:

$$
\begin{aligned}
& \int_{0}^{h_{m}} \Gamma_{1} \mathrm{~d} y_{1}=\int_{0}^{h_{m}}\left[\int_{0}^{y_{1}}\left(\frac{1}{\eta_{1}} \int_{0}^{y_{1}} \Gamma_{d}^{2} \mathrm{~d} y_{1}\right) \mathrm{d} y_{1}\right] \mathrm{d} y_{1}-\left(\int_{0}^{h_{m}} \Gamma_{m} \mathrm{~d} y_{1}\right)\left[\int_{0}^{h_{m}}\left(\frac{1}{\eta_{1}} \int_{0}^{y_{1}} \Gamma_{d}^{2} \mathrm{~d} y_{1}\right) \mathrm{d} y_{1}\right], \\
& \int_{0}^{h_{n}} \Gamma_{2} \mathrm{~d} y_{1}=\int_{0}^{h_{m}}\left[\int_{0}^{y_{1}}\left(\frac{1}{\eta_{1}} \int_{0}^{y_{1}}\left(1-\Gamma_{m}\right) \Gamma_{d} \mathrm{~d} y_{1}\right) \mathrm{d} y_{1}\right] \mathrm{d} y_{1}-\left(\int_{0}^{h_{m}} \Gamma_{m} \mathrm{~d} y_{1}\right)\left[\int_{0}^{h_{m}}\left(\frac{1}{\eta_{1}} \int_{0}^{y_{1}} \int_{0}^{y_{1}}\left(1-\Gamma_{m}\right) \Gamma_{d} \mathrm{~d} y_{1}\right) \mathrm{d} y_{1}\right] \text {, } \\
& \int_{0}^{h_{p}} \Gamma_{2} \mathrm{~d} y_{1}=\int_{0}^{h_{p}}\left[\int_{0}^{y_{1}}\left(\frac{1}{\eta_{1}} \int_{0}^{y_{1}}\left(1-\Gamma_{m}\right)^{2} \mathrm{~d} y_{1}\right) \mathrm{d} y_{1}\right] \mathrm{d} y_{1}-\left(\int_{0}^{h_{p}} \Gamma_{m} \mathrm{~d} y_{1}\right)\left[\int_{0}^{h_{m}}\left(\frac{1}{\eta_{1}} \int_{0}^{y_{1}} \int_{0}^{y_{1}}\left(1-\Gamma_{m}\right)^{2} \mathrm{~d} y_{1}\right) \mathrm{d} y_{1}\right] .
\end{aligned}
$$

The last element in Eq. (2) describes the effect of inertial forces on the pressure distribution, resulting from the variable diameter of the shaft, which disappears in the case of journal bearings.

The apparent viscosity $\eta_{p}[\mathrm{~Pa} \cdot \mathrm{s}]$ is a function of temperature, shear rate and pressure, hence the dimensionless viscosity $\eta_{1}$ [1] was modelled as the following product:

$$
\eta_{1}\left(\varphi, y_{1}, x_{1}\right)=\eta_{1 T}\left(\varphi, y_{1}, x_{1}\right) \eta_{1 \dot{\gamma}}\left(\varphi, y_{1}, x_{1}\right) \eta_{1 p}\left(\varphi, x_{1}\right) \text {. }
$$

The factor $\eta_{1 T}\left(\varphi, y_{1}, x_{1}\right)$ in Eq. (10) describes the changes in dimensionless viscosity depending on the dimensionless temperature $T_{1}=\left(T-T_{0}\right) T_{0}{ }^{-1} \mathrm{Br}^{-1}$, where $T[\mathrm{~K}]$ is dimensional temperature, $T_{0}[\mathrm{~K}]$ is the reference temperature, while dimensionless Brinkman number $[1,2,5]$ $\mathrm{Br}=U_{0}^{2} \eta_{0} \kappa_{0}^{-1} T_{0}^{-1}$, where $\kappa_{0}[\mathrm{~W} /(\mathrm{m} \cdot \mathrm{K})]$ is a characteristic value of thermal conductivity. The following model of changes in dimensionless viscosity depending on temperature was adopted $[1,2,5]$ :

$$
\eta_{1 T}\left(\varphi, y_{1}, x_{1}\right)=\exp \left(-\delta_{T} \operatorname{Br} T_{0} T_{1}\right)
$$


where $\delta_{T}\left[\mathrm{~K}^{-1}\right]$ is an experimentally determined parameter. The three-dimensional distributions of the dimensionless temperature $T_{1}\left(\varphi, y_{1}, x_{1}\right)$ in the lubrication gap of the conical slide bearing, was calculated in accordance with the following analytical solution of the energy equation $[1,6]$ :

$$
\begin{aligned}
T_{1}\left(\varphi, y_{1}, x_{1}\right)= & -\frac{1}{\kappa_{1}} \int_{0}^{y_{1}}\left(\int_{0}^{y_{1}} \Lambda_{1} \mathrm{~d} y_{1}\right) \mathrm{d} y_{1}-\frac{\cos ^{2} \gamma}{\Theta^{2} \kappa_{1}} \rho_{1}^{2} L_{1}^{2} \operatorname{Re}^{2} \psi^{2} \int_{0}^{y_{1}}\left(\int_{0}^{y_{1}} \Lambda_{2} \mathrm{~d} y_{1}\right) \mathrm{d} y_{1} \\
& +\frac{2 \cos \gamma}{\Theta \kappa_{1}} \rho_{1} L_{1} \operatorname{Re} \psi \int_{0}^{y_{1}}\left(\int_{0}^{y_{1}} \Lambda_{3} \mathrm{~d} y_{1}\right) \mathrm{d} y_{1}-\frac{q_{c 1} y_{1}}{\kappa_{1}}+1,
\end{aligned}
$$

where the dimensionless thermal conductivity $\kappa_{1}=\kappa \kappa_{0}^{-1}$ and the dimensionless heat flux:

$$
q_{c 1}=-\left.\kappa_{1} \frac{\partial T_{1}}{\partial y}\right|_{y_{1}=0} .
$$

Furthermore, the following designations were introduced in the Eq. (12):

$$
\begin{gathered}
\Lambda_{1}\left(\varphi, y_{1}, x_{1}\right)=\eta_{1}\left(\frac{1}{\Theta} \frac{\partial p_{1}}{\partial \varphi} \frac{\partial \Gamma_{d}}{\partial y_{1}}-\Theta \frac{\partial \Gamma_{m}}{\partial y_{1}}\right)^{2}+\frac{\eta_{1}}{L_{1}^{2}}\left(\frac{\partial p_{1}}{\partial x_{1}} \frac{\partial \Gamma_{d}}{\partial y_{1}}\right)^{2}, \\
\Lambda_{2}\left(\varphi, y_{1}, x_{1}\right)=\eta_{1}\left[\left(\frac{1}{\Theta} \frac{\partial p_{1}}{\partial \varphi}\right)^{2} \frac{\partial \Gamma_{1}}{\partial y_{1}}+2 \frac{\partial p_{1}}{\partial \varphi} \frac{\partial \Gamma_{2}}{\partial y_{1}}+\Theta^{2} \frac{\partial \Gamma_{3}}{\partial y_{1}}\right]^{2}, \\
\Lambda_{3}\left(\varphi, y_{1}, x_{1}\right)=\eta_{1} \frac{\partial p_{1}}{\partial x_{1}} \frac{\partial \Gamma_{d}}{\partial y_{1}}\left[\left(\frac{1}{\Theta} \frac{\partial p_{1}}{\partial \varphi}\right)^{2} \frac{\partial \Gamma_{1}}{\partial y_{1}}+2 \frac{\partial p_{1}}{\partial \varphi} \frac{\partial \Gamma_{2}}{\partial y_{1}}+\Theta^{2} \frac{\partial \Gamma_{3}}{\partial y_{1}}\right]
\end{gathered}
$$

The factor $\eta_{1 \dot{\gamma}}\left(\varphi, y_{1}, x_{1}\right)$ in Eq. (10) describes the effect of shear rate on dimensionless viscosity of generalized non-Newtonian oil. The Cross model was adopted in this study, therefore:

$$
\eta_{1 \dot{\gamma}}\left(\varphi, y_{1}, x_{1}\right)=\eta_{1 z e r o}\left[1+\left(k_{\dot{\gamma}} \dot{\gamma}\right)^{n_{\dot{\gamma}}}\right]^{-1},
$$

where $\eta_{\text {1zero }}[1]$ is the dimensionless viscosity for low shear rates $\dot{\gamma} \rightarrow 0$ [1/s], while $k_{\dot{\gamma}}$ [s] and $n_{\dot{\gamma}}[1]$ are experimental factors. The shear rate $\dot{\gamma}\left(\varphi, y_{1}, x_{1}\right)$ was calculated according to the relationship:

$$
\dot{\gamma}\left(\varphi, y_{1}, x_{1}\right)=\sqrt{\frac{1}{2} \sum_{i=1}^{3} \sum_{j=1}^{3} \dot{\gamma}_{i j} \dot{\gamma}_{j i}},
$$

where $\dot{\gamma}_{i j}[1 / \mathrm{s}]$ are the components of the strain tensor $[1,2,5]: \mathbf{A}_{1}=\mathbf{L}+\mathbf{L}^{\mathbf{T}}$, for which $\mathbf{L}=\operatorname{grad} \vec{v}$.

The dimensionless velocity component $v_{1}=v_{\varphi} U_{0}{ }^{-1}$ in the peripheral direction and the dimensionless component $v_{3}=v_{x} L_{1} U_{0}^{-1}$ in the longitudinal direction was calculated in accordance with the analytical solutions of momentum conservation equations [1,6] (the transverse velocity component $v_{2}$ was omitted):

$$
v_{1}\left(\varphi, y_{1}, x_{1}\right)=\frac{1}{\Theta} \frac{\partial p_{1}}{\partial \varphi} \Gamma_{d}-\Theta\left(\Gamma_{m}-1\right)
$$




$$
v_{3}\left(\varphi, y_{1}, x_{1}\right)=\frac{1}{\Theta} \frac{\partial p_{1}}{\partial x_{1}} \Gamma_{d}-\rho_{1} L_{1} \operatorname{Re} \psi \frac{\cos \gamma}{\Theta} \Gamma_{c}
$$

The factor $\eta_{1 p}\left(\varphi, x_{1}\right)$ in Eq. (10) determines the effect of pressure on the dimensionless viscosity. Due to the simplifications adopted for the thin lubricating layer, the model omits changes in pressure towards the height of the lubrication gap. It was assumed, that the viscosity depends on the pressure according to the following relationship:

$$
\eta_{1 p}\left(\varphi, x_{1}\right)=\ln \left(\mathrm{e}+\delta_{p} p_{0} p_{1}\right),
$$

where e $\approx 2.718$ is the Euler's number and $\delta_{p}\left[\mathrm{~Pa}^{-1}\right]$ is an experimental factor.

The coefficients for these models were determined by fitting the above functions to the experimental data obtained for $2 \%$ ferro-oil, which is not affected by the magnetic field. These results are presented in the work [2].The fitting of the curves described with these models was made using the least squares approximation method and the lsqcurvefit package from the MATLAB software. The determined values are $Q_{\mathrm{Br}}=\delta_{T} \mathrm{Br} T=3.4224$ [1], $Q_{p}=\delta_{T} p_{0}=2.7097$ [1], $\eta_{1 \text { zero }}=2.71[1], k_{\dot{\gamma}}=0.7129[\mathrm{~s}]$ and $n_{\dot{\gamma}}=0.1064$ [1], for the reference values $T_{0}=363.0[\mathrm{~K}]$ and $\eta_{0}=0.0263[\mathrm{~Pa} \cdot \mathrm{s}]$. It was assumed, that: $\rho_{0}=950\left[\mathrm{~kg} / \mathrm{m}^{3}\right], \rho_{1}=1[1], \kappa_{0}=0.15[\mathrm{~W} /(\mathrm{m} \cdot \mathrm{K})]$, $\kappa_{1}=1[1]$ and the dimensionless constant temperature at bearing shaft surface $T_{1 c}=1$.

The MATLAB software from MathWorks was used to write code for this simulation. The iterative Newton's method [1,4] has been implemented, in which the first and second derivatives and mixed derivatives were approximated by finite differences. In the first step, the initial pressure distribution was calculated (by omitting nonlinear part in Eq. (2) and by assuming, that the dimensionless viscosity $\eta_{1}=1$ [1] for the Gümbel (half-Sommerfeld) condition [1, 3, 5], i.e. $\varphi_{k}=180^{\circ}$ ). The Eq. (21) was then used to calculate the viscosity changes caused by pressure changes. The determined pressure distribution was used to calculate the temperature distribution using the Eq. (12), and then to determine its effect on viscosity, from Eq. (11) and (10). Afterwards, using Eq. (19) and (20), the velocity components were calculated, which were then used to determine the shear rate distribution using Eq. (18) and its effect on the viscosity, using Eq. (17). Using the updated viscosity values, pressure corrections were calculated with the Newton's method (solution of Eq. (2)), and a new pressure distribution was determined. This procedure was repeated until the condition for convergence and residuum was reached, i.e. $<10^{-6}$. Then it was checked if the Reynold's condition was met. If not, then the $\varphi_{k}$ value, of the angle determining the end of the lubricating wedge, was increased by $\Delta \varphi_{k}=1^{\circ}$.

The obtained final pressure distribution was used to calculate the transverse $C_{1 t}[1]$ and the longitudinal $C_{1 l}$ [1] dimensionless component of the bearing load carrying capacity, with the following relations $[1,6]$ :

$$
\begin{gathered}
C_{1 t}=\sqrt{\left(\int_{-1}^{1} \int_{0}^{\varphi_{k}} p_{1} \Theta \cos \varphi \sin \gamma \mathrm{d} \varphi \mathrm{d} x_{1}\right)^{2}+\left(\int_{-1}^{1} \int_{0}^{\varphi_{k}} p_{1} \Theta \sin \varphi \sin \gamma \mathrm{d} \varphi \mathrm{d} x_{1}\right)^{2}}, \\
C_{1 t}=\sqrt{\left(\int_{-1}^{1} \int_{0}^{\varphi_{k}} p_{1} \Theta \cos \varphi \cos \gamma \mathrm{d} \varphi \mathrm{d} x_{1}\right)^{2}+\left(\int_{-1}^{1} \int_{0}^{\varphi_{k}} p_{1} \Theta \sin \varphi \cos \gamma \mathrm{d} \varphi \mathrm{d} x_{1}\right)^{2}} .
\end{gathered}
$$

The dimensionless friction force $F r_{1}$ [1] was calculated with the following equation [1]:

$$
F r_{1}=\sqrt{\left(\left.\int_{-1}^{1} \int_{0}^{2 \pi} \eta_{1} \Theta \frac{\partial v_{1}}{\partial y_{1}}\right|_{y_{1}=h_{p 1}} \mathrm{~d} \varphi \mathrm{d} x_{1}\right)^{2}+\left(\left.\int_{-1}^{1} \int_{0}^{2 \pi} \eta_{1} \Theta \frac{\partial v_{3}}{\partial y_{1}}\right|_{y_{1}=h_{p 1}} \mathrm{~d} \varphi \mathrm{d} x_{1}\right)^{2}}
$$

and the coefficient of friction $\mu_{r}[1]$ can be calculated as $[1,5,6]$ : 


$$
\mu_{r}=F r_{1}^{-1} \sqrt{C_{1 t}^{2}+C_{1 l}^{2}} .
$$

The simulations were carried out for the bearing with $L_{1}=0.5$ [1], $R_{0}=0.025$ [m], $\psi=10^{-3}[1]$, $\gamma=60^{\circ}$ and $\omega=5000[\mathrm{rpm}]$, at relative eccentricities $\lambda=0.1-0.9$, for the dimensionless heat fluxes at the bearing shaft surface $q_{c 1}=-1.0,-0.5,0.0,0.5,1.0$. The negative values of $q_{c 1}$ indicate that the heat is flowing away from the lubricating gap, and the positive values of $q_{c 1}$ mean, that the heat is flowing into the lubricant.

\section{Results and discussion}

Table 1 shows the influence of the modelled heat flux at the bearing shaft surface, on the generated value of the transverse component $C_{t}[1]$ of the dimensionless bearing load carrying capacity. The reference values are the results for the case, when the dimensionless heat flux (at the shaft surface) $q_{c 1}=0.0$ [1]. In other cases (that is for the $q_{c 1}=-1.0,-0.5,0.5,1.0$ ), the relative changes in the transverse component of load carrying capacity, for various values of the heat flux at the shaft surface, were calculated with accordance to the following formula:

$$
\delta X=\left(X_{1}-X_{r}\right) X_{r}^{-1} \cdot 100 \%,
$$

where $X_{r}$ is the reference value (for $q_{c 1}=0.0$ [1]) and $X_{1}$ is the considered quantity.

Tab. 1. The influence of the heat flux at the bearing shaft on the transverse component of the load carrying capacity

\begin{tabular}{|c|c|c|c|c|c|}
\hline \multirow{2}{*}{$\lambda[1]$} & \multicolumn{2}{|c|}{$\delta C_{t}[\%]$} & $C_{t}[1]$ & \multicolumn{2}{c|}{$\delta C_{t}[\%]$} \\
\cline { 2 - 6 } & $q_{c 1}=-1.0[1]$ & $q_{c 1}=-0.5[1]$ & $q_{c 1}=0.0[1]$ & $q_{c 1}=0.5[1]$ & $q_{c 1}=1.0[1]$ \\
\hline 0.1 & -3.4 & -2.5 & 0.012 & 2.5 & 5.0 \\
\hline 0.2 & -2.7 & -2.3 & 0.026 & 2.3 & 5.0 \\
\hline 0.3 & -2.8 & -2.1 & 0.043 & 2.6 & 5.1 \\
\hline 0.4 & -2.7 & -2.1 & 0.067 & 2.5 & 4.9 \\
\hline 0.5 & -2.7 & -2.0 & 0.103 & 2.5 & 5.1 \\
\hline 0.6 & -2.8 & -2.1 & 0.164 & 2.5 & 5.1 \\
\hline 0.7 & -2.8 & -2.1 & 0.285 & 2.6 & 5.2 \\
\hline 0.8 & -3.0 & -2.3 & 0.599 & 2.7 & 5.5 \\
\hline 0.9 & -3.3 & -2.6 & 2.134 & 3.1 & 6.3 \\
\hline
\end{tabular}

When assuming in simulations, that the heat flux at the shaft surface is negative, reduction of the transverse load carrying capacity component was obtained. For $q_{c 1}=-0.5$ [1], the relative decrease was from $2.0[\%]$ at $\lambda=0.5$, to $2.6[\%]$ at $\lambda=0.9$. For $q_{c 1}=-1.0$ [1], the relative decrease was from $2.6[\%]$ at $\lambda=0.4$, to $3.3[\%]$ at $\lambda=0.9$. In the calculations carried out for the cases, where heat flows from the bearing shaft to the lubrication gap, the $C_{t}$ value increased. For $q_{c 1}=0.5$ [1], they were increments of 2.5-3.1 [\%]. Increasing the heat flux to $q_{c 1}=1.0$ [1] caused more significant relative changes than when increasing the value of heat flowing out from $q_{c 1}=-0.5$ [1] to $q_{c 1}=-1.0$ [1]. At $q_{c 1}=1.0$ [1], the increments of transverse component of bearing load carrying capacity were of 4.3-6.3 [\%].

In Tab. 2 are presented the results for the longitudinal component $C_{l}$ [1] of the dimensionless bearing load carrying capacity. The influence of the heat flux at the bearing shaft surface, on the generated friction forces is presented in Tab. 3 .

The heat flux at the bearing shaft surface $q_{c 1}$ does not have such a significant effect on friction forces, in relation to the induced changes in load carrying capacities. The relative changes in the friction force exceeded $1[\%]$ only for a few of the investigated cases and reached the highest value of 1.9 [\%] for $q_{c 1}=1.0$ [1], at $\lambda=0.9$. How the value of $q_{c 1}$ affects the coefficient of friction of the bearing, calculated with the Eq. (25), is shown in the Tab. 4. 
The presented results show, that the assumption of heat flowing away from the lubrication gap causes the reduction of bearing load carrying capacities and friction forces, whereas when simulations assume, that the heat flows from the surface of the shaft to the lubricating oil, it causes an increase in the bearing load carrying capacities and friction forces. The relative changes are greater for the load carrying capacities than for the friction forces, which in effect, causes an increase in the value of the coefficient of friction for the negative $q_{c 1}$, and its decrease for the positive $q_{c 1}$, in relation to the $q_{c 1}=0.0$ [1].

Tab. 2. The influence of the heat flux at the bearing shaft on the longitudinal component of the load carrying capacity

\begin{tabular}{|c|c|c|c|c|c|}
\hline \multirow{2}{*}{$\lambda[1]$} & \multicolumn{2}{|c|}{$\delta C_{l}[\%]$} & $C_{l}[1]$ & \multicolumn{2}{c|}{$\delta C_{l}[\%]$} \\
\cline { 2 - 6 } & $q_{c 1}=-1.0[1]$ & $q_{c 1}=-0.5[1]$ & $q_{c 1}=0.0[1]$ & $q_{c 1}=0.5[1]$ & $q_{c 1}=1.0[1]$ \\
\hline 0.1 & -2.9 & -2.9 & 0.007 & 2.4 & 4.3 \\
\hline 0.2 & -2.7 & -2.0 & 0.015 & 2.7 & 4.7 \\
\hline 0.3 & -2.8 & -2.0 & 0.025 & 2.4 & 4.8 \\
\hline 0.4 & -2.6 & -2.1 & 0.039 & 2.6 & 5.2 \\
\hline 0.5 & -2.7 & -2.0 & 0.059 & 2.5 & 5.1 \\
\hline 0.6 & -2.9 & -2.1 & 0.095 & 2.4 & 5.1 \\
\hline 0.7 & -2.9 & -2.1 & 0.164 & 2.6 & 5.2 \\
\hline 0.8 & -3.0 & -2.3 & 0.346 & 2.7 & 5.5 \\
\hline 0.9 & -3.3 & -2.6 & 1.232 & 3.1 & 6.3 \\
\hline
\end{tabular}

Tab. 3. The influence of the heat flux at the bearing shaft surface on the friction force

\begin{tabular}{|c|c|c|c|c|c|}
\hline \multirow{2}{*}{$\lambda[1]$} & \multicolumn{2}{|c|}{$\delta F_{t}[\%]$} & $F_{t}[1]$ & \multicolumn{2}{c|}{$\delta F_{t}[\%]$} \\
\cline { 2 - 6 } & $q_{c 1}=-1.0[1]$ & $q_{c 1}=-0.5[1]$ & $q_{c 1}=0.0[1]$ & $q_{c 1}=0.5[1]$ & $q_{c 1}=1.0[1]$ \\
\hline 0.1 & -0.5 & -0.4 & 7.0 & 0.4 & 0.9 \\
\hline 0.2 & -0.5 & -0.4 & 7.0 & 0.5 & 0.9 \\
\hline 0.3 & -0.5 & -0.4 & 7.1 & 0.5 & 1.0 \\
\hline 0.4 & -0.6 & -0.5 & 7.2 & 0.5 & 1.0 \\
\hline 0.5 & -0.6 & -0.5 & 7.5 & 0.5 & 1.0 \\
\hline 0.6 & -0.6 & -0.5 & 7.9 & 0.5 & 1.1 \\
\hline 0.7 & -0.7 & -0.5 & 8.6 & 0.6 & 1.2 \\
\hline 0.8 & -0.8 & -0.6 & 9.8 & 0.7 & 1.4 \\
\hline 0.9 & -1.1 & -0.9 & 13.1 & 1.0 & 1.9 \\
\hline
\end{tabular}

Tab. 4. The influence of the heat flux at the bearing shaft surface on the coefficient of friction

\begin{tabular}{|c|c|c|c|c|c|}
\hline \multirow{2}{*}{$\lambda[1]$} & \multicolumn{2}{|c|}{$\delta \mu_{t}[\%]$} & $\mu_{t}[1]$ & \multicolumn{2}{c|}{$\delta \mu_{t}[\%]$} \\
\cline { 2 - 6 } & $q_{c 1}=-1.0[1]$ & $q_{c 1}=-0.5[1]$ & $q_{c 1}=0.0[1]$ & $q_{c 1}=0.5[1]$ & $q_{c 1}=1.0[1]$ \\
\hline 0.1 & 2.4 & 1.8 & 507 & -2.1 & -3.9 \\
\hline 0.2 & 2.3 & 1.7 & 235 & -2.0 & -3.9 \\
\hline 0.3 & 2.3 & 1.7 & 142 & -1.9 & -3.8 \\
\hline 0.4 & 2.3 & 1.7 & 94 & -1.9 & -3.8 \\
\hline 0.5 & 2.2 & 1.6 & 63 & -1.9 & -3.8 \\
\hline 0.6 & 2.2 & 1.6 & 42 & -1.9 & -3.8 \\
\hline 0.7 & 2.2 & 1.6 & 26 & -1.9 & -3.8 \\
\hline 0.8 & 2.2 & 1.7 & 14 & -2.0 & -3.9 \\
\hline 0.9 & 2.2 & 1.7 & 5 & -2.1 & -4.1 \\
\hline
\end{tabular}


The changes in the calculated values of operating parameters result directly from changes in viscosity values due to the temperature distribution depending on the heat flux $q_{c 1}$. The simulations, in which $q_{c 1}$ was positive, i.e. in which heat was supplied to the lubricating oil from the shaft surface, in a non-intuitive manner, resulted in a reduction of the average oil dimensionless temperature in the lubrication gap, as shown in Fig. 2a. The heat transfer from the lubricating oil to the surface of the shaft, caused that the average dimensionless temperature in the lubrication gap was higher than in the case when the heat flux $q_{c 1}=0.0$.
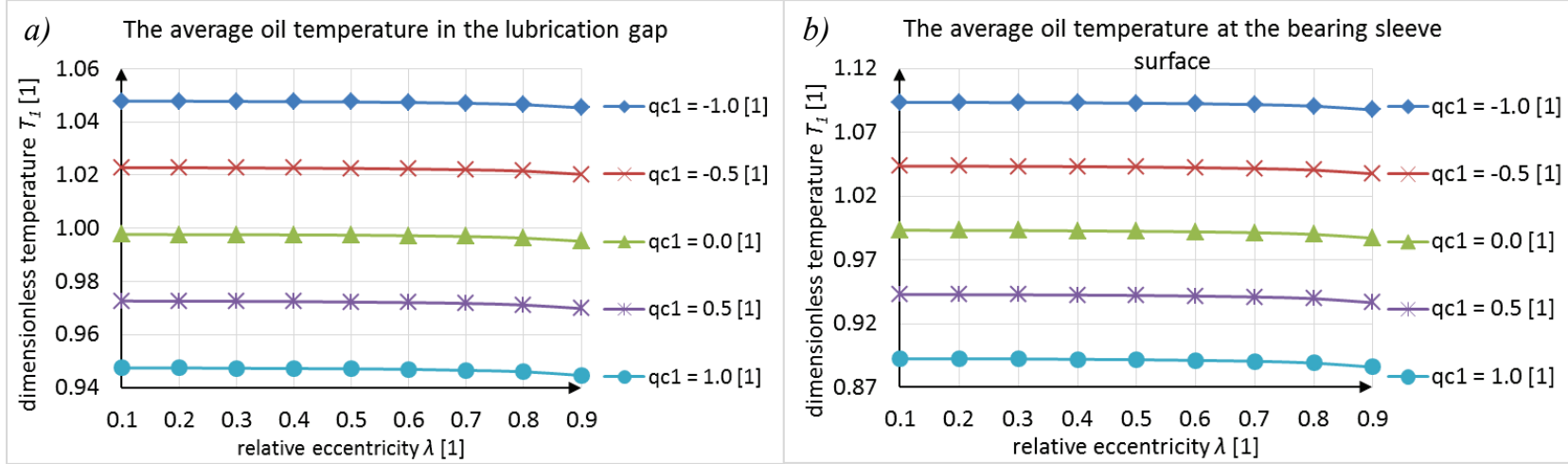

Fig. 2. The average oil temperature in the bearing lubrication gap, for the investigated values of heat flux exchanged with the shaft surface, at the relative eccentricities $\lambda=0.1-0.9$

The considered case of stationary lubrication is characterized by constancy of all parameters with respect to time; therefore, the total heat supplied on the surface of the shaft and generated as a result of dissipation inside the lubricating oil, must be equal to the value of heat flowing out of the lubrication gap. In the adopted model, this can only take place at the bearing sleeve. The more heat comes from the shaft surface, the more of heat must flow to the sleeve surface. Assuming a stationary process, a constant value of the thermal conduction coefficient of the oil and a constant distribution of the lubrication gap height, the only parameter influencing the modelled constant heat flux is the oil-calculated temperature. In particular, the average temperature of the oil layer at the bearing sleeve is shown in Fig. 2 b.

In the case when the value of the heat flowing from the lubricating oil to the surface of the shaft is greater than the heat generated as a result of dissipation, then for the assumed model, the heat must flow from the surface of the sleeve. This will happen, if the temperature at the sleeve surface is higher than the constant temperature at the shaft surface, hence the average values of dimensionless temperatures in the lubrication gap and at the sleeve surface are greater than 1 .

\section{Conclusions}

1. The adopted hydrodynamic lubrication model can be used for stationary lubrication of the conical slide bearings, when a constant temperature and heat flux at bearing shaft surface are known.

2. Assuming that the heat flows into the oil gap from the bearing shaft, cause an increase in the calculated values of load carrying capacities and friction forces, while reduces the coefficient of friction, because the average temperature of the oil in the lubrication gap decreases, which increases the viscosity; the reverse situation arises, when the heat flows out of the lubrication gap to the surface of the bearing shaft.

3. Increasing the amount of heat flowing from the lubricating gap to the shaft, from $q_{c 1}=-0.5$ [1] to $q_{c 1}=-1.0$ [1], was not as significant as the case, when the amount of heat flowing into the oil from the shaft was increased from $q_{c 1}=0.5$ [1] to $q_{c 1}=1.0$ [1]. 
4. The use of analytical solutions of simplified equations of momentum and energy causes, that in simulations, there is no need numerically solve systems of equations, in order to determine the components of velocity vector and temperature values.

\section{References}

[1] Czaban, A., Analiza hydrodynamicznego smarowania ferroolejami stożkowego łożyska ślizgowego, Rozprawa dokt., Uniwersytet Morski, Gdynia 2019.

[2] Frycz, M., Wplyw stężenia cząstek magnetycznych $w$ ferro-oleju na parametry przepływowe i eksploatacyjne poprzecznych łożysk ślizgowych, Rozprawa doktorska, Akademia Morska, Gdynia 2018.

[3] Gohar, R., Elastohydrodynamics, Imperial College Press, Singapore 2001.

[4] Kelley, C. T., Iterative Solution of Systems of Linear and Nonlinear Equations, Society for Industrial and Applied Mathematics, Philadelphia 1995.

[5] Miszczak, A., Analiza hydrodynamicznego smarowania ferrociecza poprzecznych łożysk ślizgowych, Fundacja Rozwoju Akademii Morskiej, Gdynia 2006.

[6] Wierzcholski, K., Miszczak, A., Mathematical principles and methods of biological surface lubrication with phospholipids bilayers, Biosystems, Vol. 178, pp. 32-40, 2019.

Manuscript received 16 September 2019; approved for printing 12 December 2019 